\title{
Finite difference model of a four-electrode conductivity measurement system
}

\author{
Noel D. Montgomery ${ }^{1,2,4}$, Jack L. Lancaster ${ }^{3}$ \\ 1. Air Force Research Laboratory, Bioeffects Division, Fort Sam Houston, TX \\ 2. Joint UTSA/UTHSCSA Biomedical Engineering Program, San Antonio, TX. \\ 3. Research Imaging Institute, University of Texas Health Science Center at San Antonio, San Antonio, TX \\ 4. E-mail any correspondence to: noel.montgomery@yahoo.com
}

\begin{abstract}
A finite difference model of a four-electrode tissue conductivity measurement system was developed and shown to be within $10 \%$ of theory. The model is useful for explaining the behavior of conductivity measurement electrodes in tissue.
\end{abstract}

Keywords: Conductivity measurement, current, voltage, electrodes

\section{Introduction}

Four electrode measurement (4EM) systems have been used to measure conductivity in tissue where electrode polarization would cause unacceptable inaccuracy in measurement results and where directional measurement of conductivity is required $^{1}(1-3)$. The system described here is intended for use in testing conductivity in brain tissues in vitro.

The response of the 4EM has been well characterized in homogeneous, isotropic media as $(1,3)$ :

$$
\mathrm{V}=\frac{I}{4 \pi \sigma a}
$$

Equation (1) assumes the four electrodes are linearly arranged with two high impedance potential measurement electrodes $(\mathrm{V} 1, \mathrm{~V} 2)$ flanked by two current injection electrodes (I1,I2). It also assumes the electrodes have uniform spacing $(a)$ and are surrounded by a medium with conductivity $\sigma$. The injected current is from a point source (I1) and is collected by a point sink (I2) (Figure 1).

In addition to the response function (Equation 1), Robillard and Poussart (4) also characterized the field of view of the 4EM system as approximately $3 a$ in homogeneous isotropic media. In other words, conductivity differences in media outside a radius of $3 a$ from any of the electrodes should have little effect on the response.

Steendijk (5) found solutions for 4EM systems for several conductivity environments. Starting with the infinite space of isotropic material solution (1) (Equation 1), he found a solution for an infinite half space with electrodes placed on the air/media boundary:

$$
V=\frac{I}{2 \pi \sigma a}
$$

He also found equations for an infinite half space of anisotropic material, longitudinal (long) and transverse (trans) to the fiber direction:

$$
\begin{gathered}
V_{\text {si,long }}=\frac{I}{2 \pi a \sigma_{\text {trans }}} \\
V_{\text {si,trans }}=\frac{I}{2 \pi a} \sqrt{\frac{1}{\sigma_{\text {trans }}} \frac{1}{\sigma_{\text {long }}}}
\end{gathered}
$$

Steendijk's solutions only hold for specific geometries. A real world measurement in anisotropic or mixed tissue would necessarily require a much more complex solution, if such a solution is even possible.

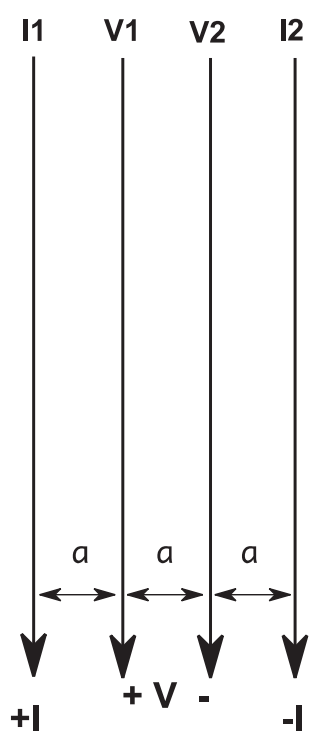

Figure 1. Electrode configuration of a 4EM system.

\footnotetext{
${ }^{1}$ Less polarization than a two-electrode measurement system where the current is applied by the same electrodes that measures the potential and directional measurement as opposed to a coaxial measurement system that measures bulk conductivity but gives no information about direction.
} 
This paper will demonstrate a finite difference numerical solution based on a three dimensional matrix of conductivity tensors to support any combination of included regions, limited only by the resolution and size of the grid. The tradeoff between either a finer grid size or increased spatial resolution is processing time.

\section{Materials and methods}

Dey and Morrison (6) developed a finite difference solution for regions of differing isotropic conductivity in geological media. This paper will extend their solution to allow differing anisotropic regions. The solution begins with a few basic equations:

$$
\begin{gathered}
J=\sigma E \\
E=-\nabla \phi \\
\nabla \cdot J=\frac{\partial \rho}{\partial t} \delta(x y z)
\end{gathered}
$$

Equation (4) is the differential form of Ohm's law that relates current density $(\mathrm{J})$ in a medium of conductivity $\sigma$ to the electric field (E) in the medium. Equation (5) holds because the field is irrotational so $\mathrm{E}$ is the gradient of the potential $(\phi)$, and equation (6) relates the time derivative of a point charge $(\rho)$ at location xyz to the divergence of $\mathrm{J}$.

Combining these equations yields:

$$
-\nabla \cdot\left[\sigma_{x y z} \nabla \phi_{x y z}\right]=\frac{\partial \rho}{\partial t} \delta(x y z)
$$

where the quantities $\sigma_{x y z}$ and $\phi_{x y z}$ are conductivity and potential at point xyz.

The derivative of charge with respect to time is equivalent to the current within a volume element: $\frac{\partial \rho}{\partial \mathrm{t}}=\frac{\mathrm{I}}{\Delta \mathrm{V}}$. Integrating both sides over the volume element $(d V)$ :

$$
-\iiint_{\Delta V} \nabla \cdot\left[\sigma_{x y z} \nabla \phi_{x y z}\right] d V=\iiint_{\Delta V} \frac{I}{\Delta V} \delta(x y z) d V
$$

The left hand side of Equation (8) can be converted to a surface integral using Green's theorem:

$$
\begin{aligned}
-\iiint_{\Delta V} \nabla \cdot\left[\sigma_{\mathrm{xyz}} \nabla\right. & \left.\phi_{\mathrm{xyz}}\right] \mathrm{dV} \\
& =-\iint_{\mathrm{S}} \sigma \nabla \phi \cdot \mathrm{ndA} \\
& =-\iint_{\mathrm{S}} \sigma \frac{\partial \phi}{\partial \mathrm{n}} \mathrm{dA}
\end{aligned}
$$

where $\mathrm{dA}$ is the surface area element.
And the right hand side of Equation (8) is simply equivalent to the current at the point $\mathrm{xyz}, \mathrm{I}_{\mathrm{xyz}}$. The resulting equation can be discretized:

$$
-\iint_{\mathrm{s}} \sigma \frac{\partial \phi}{\partial \mathrm{n}} \mathrm{dA}=\mathrm{I}_{\mathrm{xyz}}
$$

To simplify the equations, we utilize a uniform spatial grid throughout the matrix where the voxels have the same dimension in each direction, $\Delta \mathrm{x}=\Delta \mathrm{y}=\Delta \mathrm{z}=\mathrm{h}$. The lefthand side of Equation (10) is integrated by breaking into separate equations for each face and using the finite difference approximation for the normal derivative.

Dey and Morrison estimated the conductivity at each voxel face by averaging the conductivities of four adjacent voxels. In order to account for anisotropic conductivities, we used conductivity of the adjacent cell in a direction normal to the face being integrated $\left(\sigma_{\text {norm }}\right)$. This should introduce minimal error as long as the change in conductivity is gradual local to the voxel. Care should be taken in interpreting the potentials immediately adjacent to a boundary between two conductivities.

The discretized solutions for each face are summed to comprise the surface integral:

$$
-\sum_{\text {face }} \sigma_{\text {norm }}\left(\frac{\phi_{\text {adj }}-\phi_{\text {self }}}{\mathrm{h}}\right) h^{2}
$$

where:

$$
\iint_{S} \mathrm{dA}=\mathrm{h}^{2}
$$

and the values of the conductivity $\left(\sigma_{a d j}\right)$ and potential $\left(\phi_{a d j}\right)$ of the adjacent voxel are defined as in Figure 2. Coupling constants as defined by Dey and Morrison (6) are developed for each face and for the object voxel, refer to Figure (2) for definition of conductivity for each face:

$$
\begin{gathered}
C_{1}=\sigma_{1} * h \\
C_{2}=\sigma_{2} * h \\
C_{3}=\sigma_{3} * h \\
C_{4}=\sigma_{4} * h \\
C_{5}=\sigma_{5} * h \\
C_{6}=\sigma_{6} * h \\
C_{\text {self }}=-\left(C_{1}+C_{2}+C_{3}+C_{4}+C_{5}+C_{6}\right)
\end{gathered}
$$

All the analysis to this point applies to voxels in the interior of the volume of a conductive region. Because the size of the region is necessarily limited, some attention needs to be paid to the behavior at the limits of the computational volume. Voxels at the boundary of the volume require a boundary 
condition to allow accurate prediction of potentials across the boundary.

The simplest boundary condition is to assume the potential at the boundaries is a constant (usually zero), as if the boundaries are at infinite distance from the probe (Dirichlet boundary condition). Implementation of the Dirichlet boundary condition resulted in an overprediction of the measured potentials by $25 \%$ for anisotropic cases, presumably due to interactions of fields with finite boundaries. Although Robillard and Poussart (4) found a field of view of $3 a$ in an isotropic medium, an anisotropic medium may alter the field of view by extending the range of the currents in the low conductivity direction.

A mixed boundary condition:

$$
\alpha \phi+\beta \frac{\partial \phi}{\partial \mathrm{n}}=\mathrm{f}(\mathrm{x}, \mathrm{y}, \mathrm{z})
$$

may yield a more accurate prediction. The field at a distance (r) from the point current source behaves as $\phi \propto \frac{1}{r}$, so the boundary condition becomes:

$$
\frac{\partial \phi}{\partial \mathrm{n}} \propto-\frac{1}{\mathrm{r}^{2}} \hat{\mathrm{e}}_{\mathrm{r}} \cdot \hat{\mathrm{n}}
$$

where $\hat{\mathrm{e}}_{\mathrm{r}}$ is the unit vector in the direction from the point current source to the boundary location and $\hat{n}$ is the unit vector normal to the face at the boundary. $\hat{e} \cdot \hat{r}=r \cos (\theta)$ so the boundary condition is:

$$
\alpha \phi+\beta \frac{\phi \cos (\theta)}{r}=f(x, y, z)
$$

where $\cos (\theta)$ is the $\operatorname{cosine}$ of the angle between the vector from the point current source to the boundary voxel and the normal direction of the face on the boundary. And $r$ is the distance from the source to the voxel. This definition greatly complicates the execution of the model because creation of a new finite difference matrix is necessary for each source location. To simplify model creation, we can assume the source is always at the middle of the geometric model volume. This causes minimal error when the source is near the center of the volume (6).

As a result of the mixed boundary condition, the potential for faces on the boundary is assumed to be zero (for instance, $\mathrm{C}_{1}=0$ ) and $\mathrm{C}_{\text {self }}$ (Equation 16) gets an extra term. (Note: Face 6 is opposite Face 1).

The subscripts ctr and bnd refer to the center of the geometric model volume and the voxel on the boundary, respectively. Because the $\mathrm{C}_{\mathrm{xbnd}}$ terms are zero except for the face on the boundary, the inclusion of the extra term only slightly increases the complexity of the model.

$$
\begin{aligned}
C_{\text {self }}=-\left(C_{1}+C_{2}\right. & \left.+C_{3}+C_{4}+C_{5}+C_{6}\right) \\
& -\left(C_{1 \text { bnd }}+C_{2 \text { bnd }}+C_{3 \text { bnd }}\right. \\
& \left.+C_{4 \text { bnd }}+C_{5 \text { bnd }}+C_{6 \text { bnd }}\right)
\end{aligned}
$$

Where

$$
\mathrm{C}_{1 \mathrm{bnd}}=\mathrm{C}_{6} * \frac{\left|\mathrm{z}_{\mathrm{ctr}}-\mathrm{z}_{\mathrm{bnd}}\right|}{\sqrt{\left(\mathrm{x}_{\mathrm{ctr}}-\mathrm{x}_{\mathrm{bnd}}\right)^{2}+\left(\mathrm{y}_{\mathrm{ctr}}-\mathrm{y}_{\mathrm{bnd}}\right)^{2}+\left(\mathrm{z}_{\mathrm{ctr}}-\mathrm{z}_{\mathrm{bn}}\right.}}
$$

So the discretization of Equation (10) is:

$$
\begin{gathered}
\mathrm{C}_{1} \phi_{1}+\mathrm{C}_{2} \phi_{2}+\mathrm{C}_{3} \phi_{3}+\mathrm{C}_{4} \phi_{4}+\mathrm{C}_{5} \phi_{5}+\mathrm{C}_{6} \phi_{6} \\
+\mathrm{C}_{\mathrm{self}} \phi_{(\mathrm{i}, \mathrm{j}, \mathrm{k})}=\mathrm{I}_{\mathrm{xyz}}
\end{gathered}
$$

This equation can be written in matrix form as:

$$
[\mathrm{C}][\phi]=[\mathrm{S}]
$$

Where $[\mathrm{C}]$ is the matrix of coupling coefficients, $[\phi]$ is the vector of unknown potentials and $[S]$ is the source vector which is zero everywhere except at the location of the point current sources.

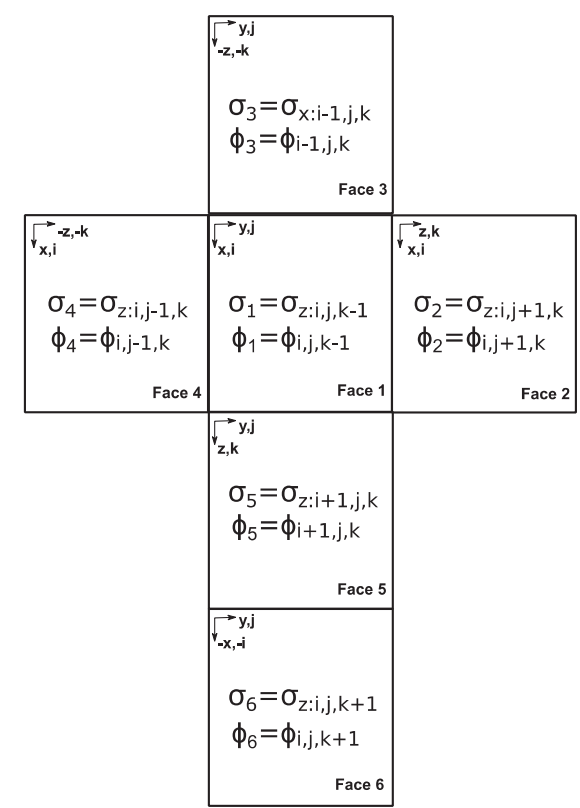

Figure 2. Orientation, conductivity, and potential of each face of a cube.

In order to construct the finite difference matrix, the 3dimensional physical matrix must be mapped to a 2dimensional matrix where each column and each row represent one of the unknown potentials. For instance, a $10 \times 10 \times 10$ cell region $(1000$ cells or voxels) would map to a 2-dimensional matrix with $1000 \times 1000$ elements representing 1000 equations with 1000 unknowns that must be solved simultaneously to determine the potential in each cell. Each row of the matrix is the equation for one unknown potential and each column element represents one unknown of the equation. 


$$
\left.\ldots \begin{array}{cccc}
C_{n-3} & C_{n-2} & C_{n-1} & C_{n} \\
& C_{n-2} & C_{n-1} & C_{n}
\end{array}\right]\left[\begin{array}{l}
\phi_{1} \\
\phi_{2}
\end{array}\right]=\left[\begin{array}{l}
S_{1} \\
S_{2}
\end{array}\right]
$$

Figure 3. Top right had corner of finite difference matrix. $\mathrm{C}$ is the multiplier of the unknown potential at each adjacent face.

Figure 3 shows the top right-hand corner of the finite difference matrix representing the system of $n$ equations and $\mathrm{n}$ unknowns where the $\mathrm{Cs}$ are the coupling coefficients with the adjacent cell. Because the potential in a cell only depends on the six adjacent potentials and its own potential, the finite difference matrix is sparse and can be manipulated using iterative techniques in Matlab (MathWorks Inc., Natick, MA) to solve for the potential in each cell. The full Matlab code is available (see bottom of page).

Several aspects of the Matlab code deserve mention. The input to the Matlab code is a geometric model consisting of a 3 dimensional array of diagonalized conductivity tensors. The implementation assumes the grid spacing to be the same in all directions $(\Delta \mathrm{x}=\Delta \mathrm{y}=\Delta \mathrm{z}=\mathrm{h})$. The largest practical size of the grid is $100 \times 100 \times 100$ voxels on a quadcore MacBook Pro. With a grid spacing of $0.1 \mathrm{~mm}$, this provides a space of $10 \mathrm{~mm} \times 10 \mathrm{~mm} \times 10 \mathrm{~mm}$ that is large enough to contain the 4EM system electrodes with a boundary of approximately $3 \mathrm{a}$. The grid is built by a custom Matlab function (GM_builder.m) using a mathematical description of the medium. The medium can have isotropic and anisotropic regions and could be imported from a diffusion tensor image to calculate the coupling coefficients if the diffusion tensor is assumed to be proportional to the conductivity tensor.

The most computationally expensive part of the code is construction of the finite difference matrix (Matlab function SetUpFDMatrix impbound.m). The matrix is built one line at a time using logical indexing and the command sub2ind that converts the three dimensional coordinates $(\mathrm{i}, \mathrm{j}, \mathrm{k})$ to a serial number $(1 \ldots \mathrm{N})$. Matlab references matrix indices in row, column, slice order so a large flat matrix can be built fairly efficiently. On a quad-core MacBook Pro this step can take from 20 minutes to one hour to complete. Fortunately, this step only needs to be accomplished once for each geometry, as it is not dependent on the location of the sources or measurement points.

The final step in predicting the response of the 4EM system is to specify the location of the point current sources (one positive and one negative) and the location of the measurement points (as shown in Figure 1). The Matlab functions SinglePoint.m and MultiPoints angulareror.m compute the potential for a single probe location or an array of probe locations within the medium. The functions take the center of the probe location and the direction of the probe axis and compute the location of each current point source and measurement location to maintain an appropriate geometry. In a physical system there could be error in the positioning of the probes, so the function MultiPoints_angularerror.m allows specification of three angular errors, $\theta_{\mathrm{x}}, \theta_{\mathrm{y}}$, and $\theta_{\mathrm{z}}$. The function can show the impact of having the probes misaligned with the interface directions on the response of the 4EM.

The solution of the finite difference equation cannot be accomplished using the straightforward Matlab backslash operator $([\phi]=[S] \backslash[C])$ due to memory limitations of the computer. Matlab offers several methods of iterative solutions for sparse matrices that make the solution possible. The General Method of Residuals (gmres) (7) is a robust method that is successful at solving the system regardless of the content of the geometric model. However, it can take up to an hour to solve the equation. The Symmetric LQ (symmLQ) (8) method is much faster for some geometries, but fails to converge if the geometries are asymmetric such as an infinite half-space. The method we selected is the Quasi-minimal residual (qmr) (9) method because it is very fast (20-40 seconds) and converges for all geometries attempted. Note that all the methods give very similar results for conditions where they converge.

\section{Results}

The finite difference model can be compared to closed form solutions like Steendijk (5). Equation (1) provides a solution for an infinite space with isotropic conductivity. Equation (4) provides solutions for electrodes on the surface of an anisotropic halfspace. Equation (4) can be modified to predict the response in an infinite anisotropic space by multiplying by a factor of $1 / 2$. As long as the distance between the outer electrodes and the boundary are more than approximately three times the electrode spacing $(3 \times a)$ the boundary will have minimal impact on the measurement.

Finite difference models are validated based on convergence to the known exact value for the solution with decreasing voxel size (h). Figure (4) shows a graph of predicted potential in an isotropic infinite space with decreasing ' $h$ ' as compared to the exact solution from Equation (1). Figures (5) and (6) show graphs of predicted potential versus ' $h$ ' for longitudinal and transverse probe orientations in an infinite anisotropic space $\left(\sigma_{l}=0.5, \sigma_{\mathrm{t}}=\right.$ 0.1 . These plots show fairly close convergence to the analytical solution with decreasing voxel size (Approximately $8 \%$ difference for the anisotropic transverse cases, approximately $2 \%$ for the other cases).

Models can be further validated by comparing them to theory over a range of values. Figures (7), (8), and (9) compare model predictions at increasing depth with theoretical predictions (Equation (4)) at the surface of an infinite half space and at depth (essentially an infinite space). Note that the finite difference model slightly

Matlab code:

https://github.com/joeb-files/2016_Montgomery 


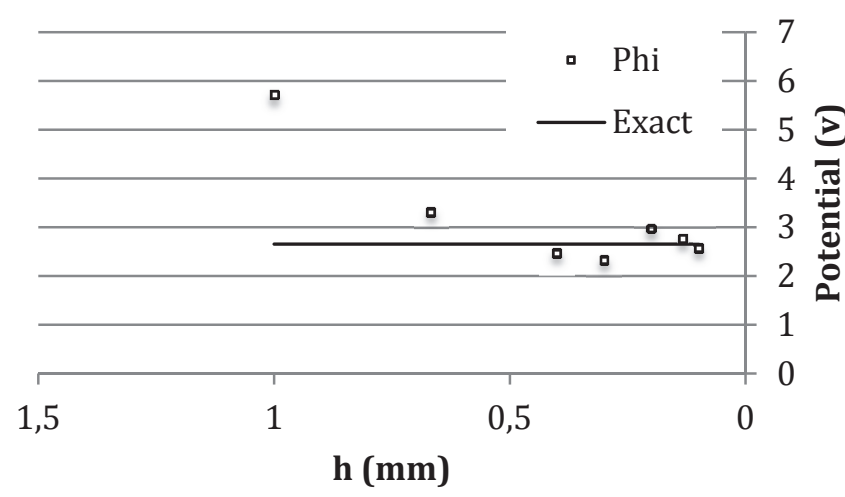

Figure 4: Convergence plot for an isotropic infinite space.

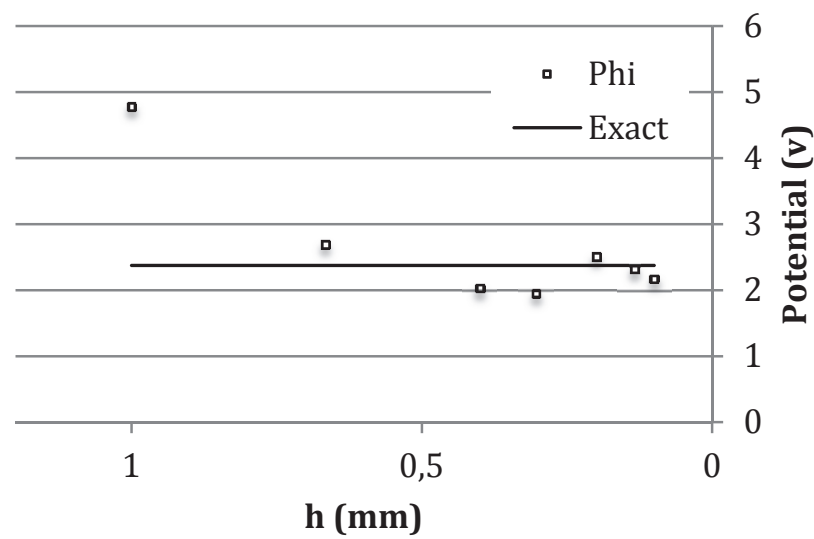

Figure 5: Convergence plot for an anisotropic infinite space, transverse direction

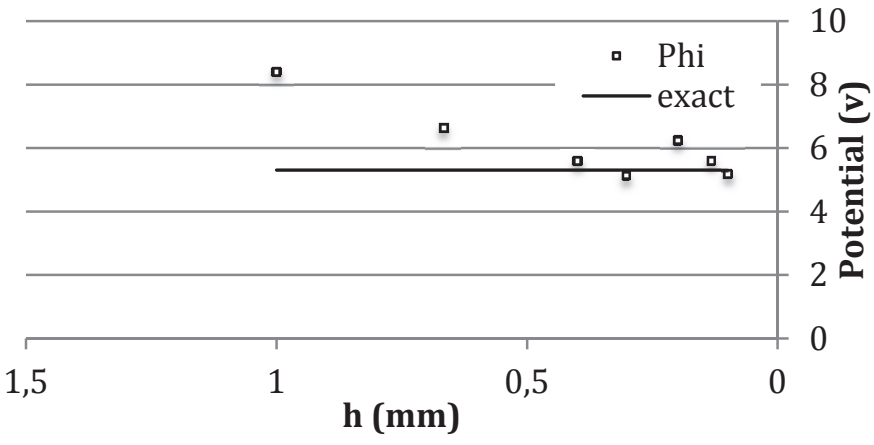

Figure 6: Convergence diagram for an anisotropic infinite space, longitudinal direction

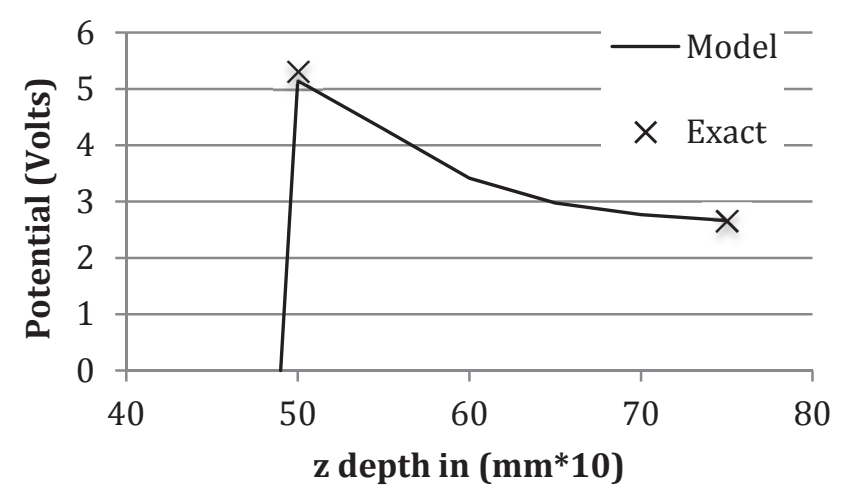

Figure 7: Potential on the surface and at depth in an isotropic infinite halfspace

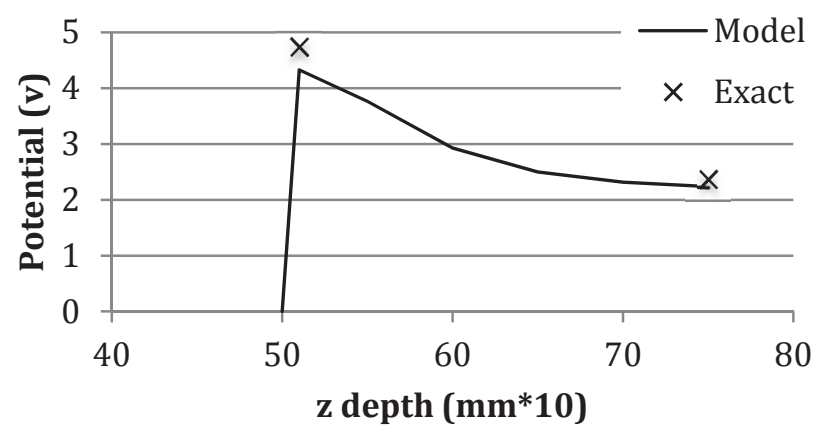

Figure 8: Potential on the surface and at depth in an anisotropic infinite halfspace, transverse direction

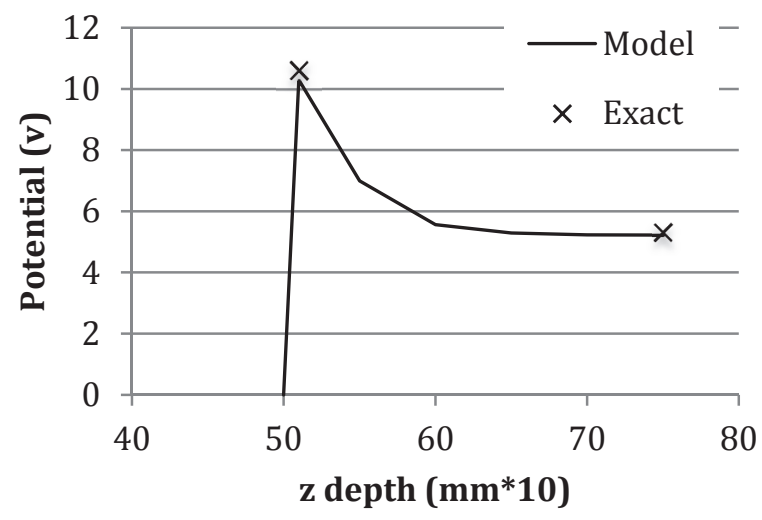

Figure 9: Potential on the surface and at depth in an anisotropic infinite halfspace, longitudinal direction

underpredicts (by $8 \%$ ) the value at the interface. This may be inaccuracy caused by the simplistic formulation (kernel) of the finite difference model. However, at depth, the model predicts within approximately $5 \%$ of the theoretical value.

Accepting that the finite difference model is a fairly good predictor of the response of the 4EM, a number of configurations can be examined. In use, the 4EM is advanced down through tissue through a layer that is thought to be anisotropic. In order to understand the response as the probes traverse the anisotropic layer, a geometry model was built with an anisotropic layer, $2 \mathrm{~mm}$ in thickness, flanked by infinite isotropic layers. Additionally, an isotropic infinite space, and an isotropic infinite halfspace will be analyzed (Figure (10)).

Figure (11) shows the predicted response of the 4EM system as the probe is traversed from 3 to $7 \mathrm{~mm}$ in depth (the $\mathrm{z}$ direction) in the center ( $\mathrm{x}$ and $\mathrm{y}$ directions) of the space. The isotropic media in these analyses have an electric conductivity of $\sigma=0.2 \mathrm{mS} / \mathrm{cm}$ and the anisotropic media have conductivities longitudinal $\sigma_{1}=0.5 \mathrm{mS} / \mathrm{cm}$, and transverse $\sigma_{\mathrm{t}}=0.1 \mathrm{mS} / \mathrm{cm}$.

The response of the 4EM system probes in the anisotropic layer is reduced by interactions with the surrounding medium as compared to the infinite case. In the transverse direction, the response is reduced by $30.2 \%$ and in the longitudinal direction the response is reduced by $20.2 \%$. 

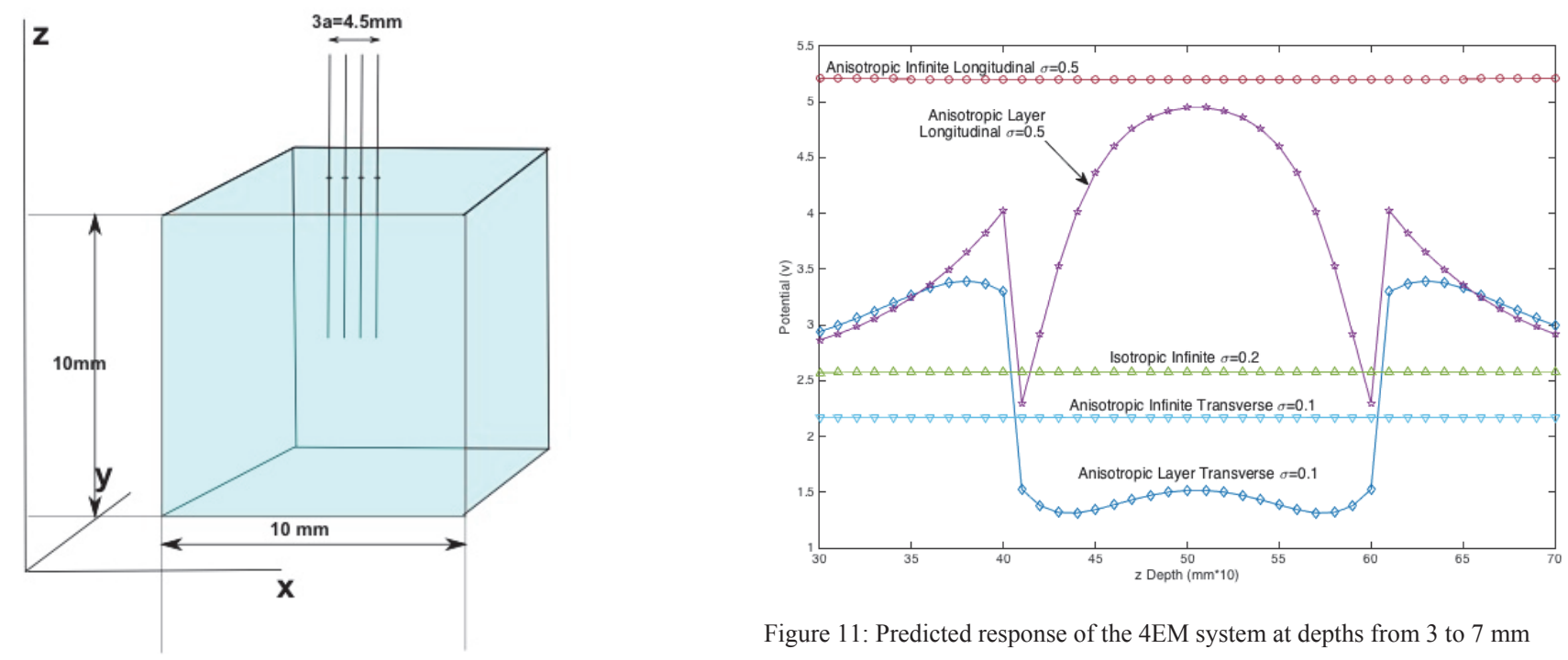

Figure 11: Predicted response of the 4EM system at depths from 3 to $7 \mathrm{~mm}$
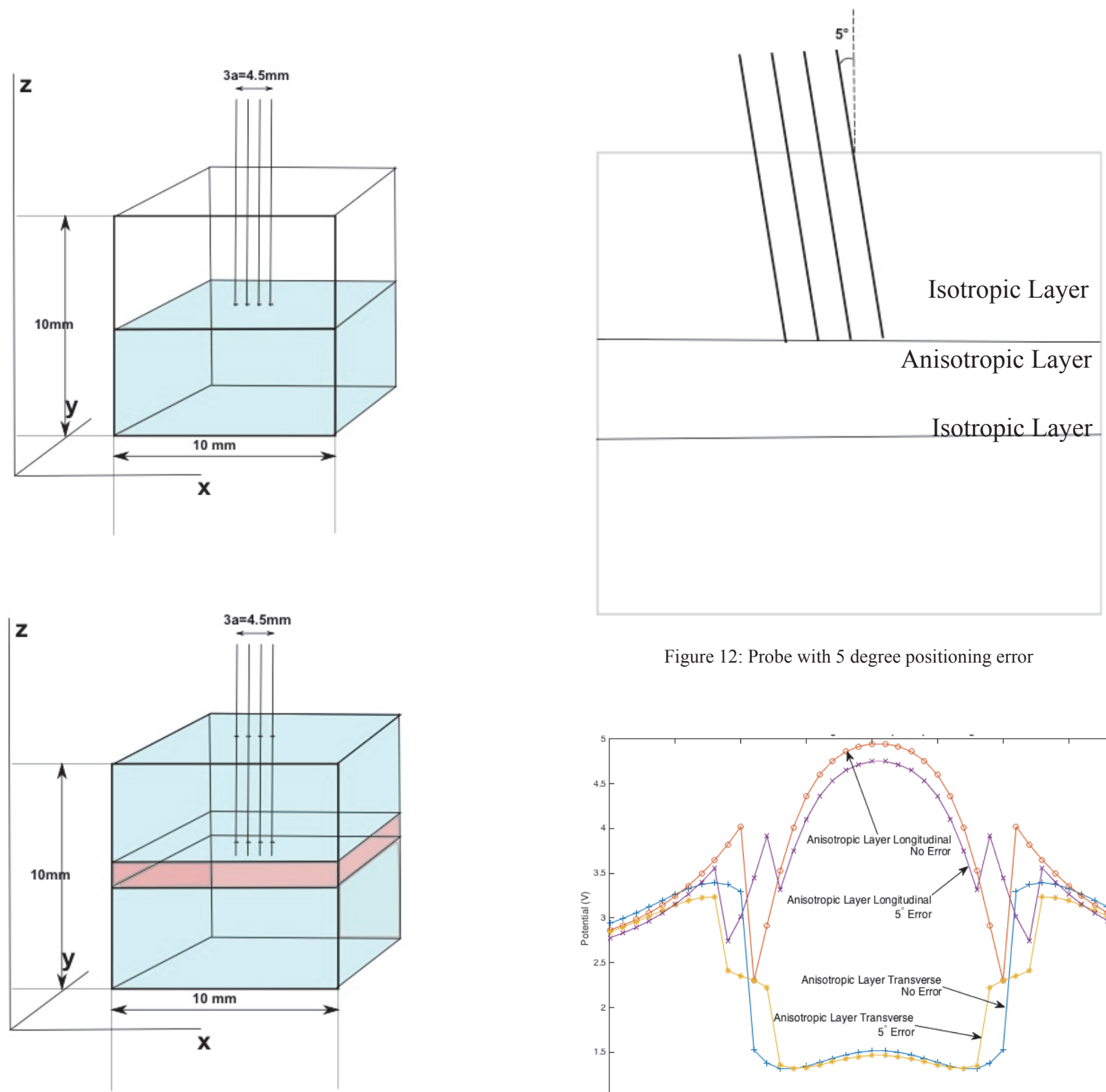

Figure 12: Probe with 5 degree positioning error

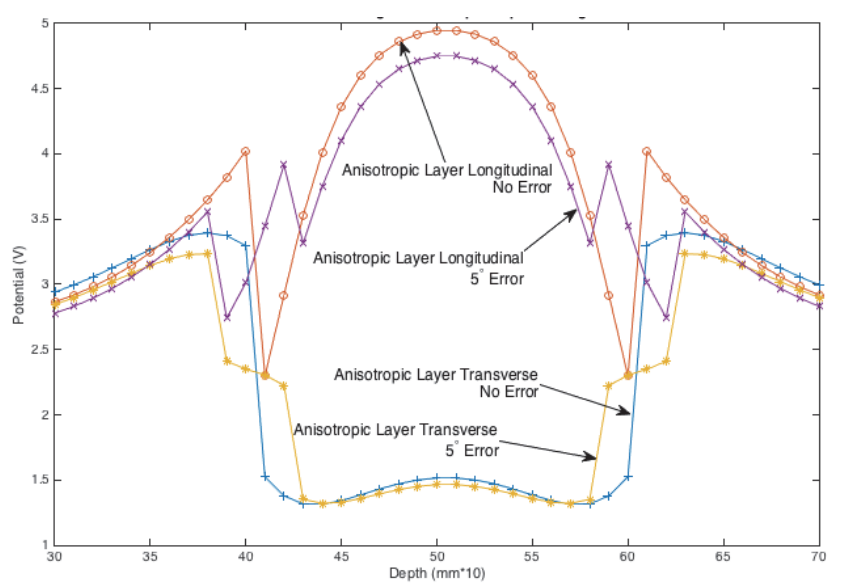

Figure 10: Geometries for analysis of 4EM system response. Top figure: infinite space. Central figure: infinite halfspace with probes on surface. Bottom figure: layer inclusion in infinite space.

Figure 13: Effect of 5 degree positioning error on 4EM system response 
Another use of the finite difference model is to explore the impact of probe positioning error on the 4EM response. Figure (12) shows the probe configuration with a 5-degree positioning error. Note that the probes enter the layer at different probe ' $z$ ' depths. The effect of this can clearly be seen in the graphs in Figure (13). The error causes the response of the $4 \mathrm{EM}$ system to be reduced by $4 \%$ in the longitudinal direction and $3.3 \%$ in the transverse direction.

\section{Discussion}

The FD model converges to the closed form result and agrees with the closed form solutions across a range of values within $10 \%$ in all cases. Furthermore, the predicted response of the 4EM system is reasonable as the probes traverse down through an anisotropic layer. Future work on this effort could include measurement of anisotropic media of known conductivity to further validate the model.

The FD model will be extremely useful to those who need to directly measure conductivity with depth in tissue if the nature of the tissue structure is known well enough to form a geometric model. It is also useful to analyze a range of tissue and geometric properties (e.g. layer thickness, anisotropy ratio) for comparison to measured data and to explain 4EM system response in anisotropic heterogeneous media. The demonstrated errors caused by a 5-degree error in probe positioning as shown in Figure 13, help provide confidence in the usefulness of the 4EM system in a practical measurement situation.

\section{References}

1. Rush S, Abildskov JA, McFee R. Resistivity of Body Tissues at Low Frequencies. Circ Res. 1963;12(1):4050. http://dx.doi.org/10.1161/01.RES.12.1.40

2. Schwan HP. Electrode polarization impedance and measurements in biological materials. Ann N Y Acad Sci. 1968;148(1):191-209. http://dx.doi.org/10.1111/j.1749-6632.1968.tb20349.x
3. Robillard PN, Poussart Y. Specific-impedance measurements of brain tissues. Medical \& biological engineering \& computing. 1977;15(4):438-45. http://dx.doi.org/10.1007/BF02457999

4. Robillard PN, Poussart D. Spatial resolution of four electrode array. IEEE transactions on bio-medical engineering. 1979;26(8):465-70. http://dx.doi.org/10.1109/TBME.1979.326572

5. Steendijk P, Mur G, Van Der Velde ET, Baan J. The four-electrode resistivity technique in anisotropic media: theoretical analysis and application on myocardial tissue in vivo. Biomedical Engineering, IEEE Transactions on. 1993;40(11):1138-48. http://dx.doi.org/10.1109/10.245632

6. Dey A, Morrison HF. Resistivity modeling for arbitrarily shaped three-dimensional structures. Geophysics. 1979;44(4):753-80. http://dx.doi.org/10.1190/1.1440975

7. Saad Y, Schultz MH. GMRES: A generalized minimal residual algorithm for solving nonsymmetric linear systems. SIAM Journal on scientific and statistical computing. 1986;7(3):856-69. http://dx.doi.org/10.1137/0907058

8. Paige CC, Saunders MA. Solution of sparse indefinite systems of linear equations. SIAM Journal on Numerical Analysis. 1975;12(4):617-29. http://dx.doi.org/10.1137/0712047

9. Freund RW, Nachtigal NM. QMR: a quasi-minimal residual method for non-Hermitian linear systems. Numerische Mathematik. 1991;60(1):315-39. http://dx.doi.org/10.1007/BF01385726 